\title{
Ultrathin and Flexible Self-powered Temperature Sensor Based on Sputtered Tellurium Nanoparticles
}

\author{
Imran Khan, ${ }^{1}$ Zong-Hong Lin, ${ }^{2,3^{*}}$ and Yu-Lin Wang ${ }^{1,3^{* *}}$ \\ ${ }^{1}$ Institute of NanoEngineering and Microsystems, National Tsing Hua University, Hsinchu 30013, Taiwan \\ ${ }^{2}$ Institute of Biomedical Engineering, National Tsing Hua University, Hsinchu 30013, Taiwan \\ ${ }^{3}$ Department of Power Mechanical Engineering, National Tsing Hua University, Hsinchu 30013, Taiwan
}

(Received December 7, 2019; accepted April 1, 2020)

Keywords: temperature detection, self-powered sensor, tellurium nanoparticles, thermoelectric effect, flexibility, ultrathin device

The energy crisis has worsened with the increase in population; to overcome this crisis, self-powered devices are required urgently. Human beings have also become increasingly health conscious and require modern equipment to monitor their body conditions. Body temperature is a measure of the thermoregulation in the body and also indicates other health conditions. For continuous body temperature monitoring, smart sensors are the best choice. In this work, we fabricate an ultrathin $(150 \mathrm{~nm})$ and flexible temperature sensor using thermoelectric tellurium nanoparticles (Te-NPs) sputtered on a thin Al substrate that can detect the exact surface temperature relative to its ambient temperature measurement. The sensor can sense both hot and cold surfaces and shows a linear response with increasing or decreasing temperature measurement. Because of its compact size and flexibility, it can conform to any surface for temperature. For a temperature gradient of $75^{\circ} \mathrm{C}$, the sensor gives an output voltage of $0.4 \mathrm{mV}$ and can even accurately measure body temperature with only a human finger touch. Such a flexible and thin device can be used in the day-to-day monitoring of the human body or any other surface whose temperature should be determined before touching it.

\section{Introduction}

The population of the world is increasing rapidly and, with the increase in population, the demand for energy is increasing. As the energy resources are limited, scientists are looking for alternate energy sources. Waste heat is present in the environment and harvesting it in the form of green renewable energy is required urgently. ${ }^{(1)}$ Nanogenerators are promising waste heat energy harvesters. Waste heat can be used as an input to drive self-powered sensors and it works on the principle of the thermoelectric effect, an effect that produces an electrical output whenever a temperature gradient is created. With the growing population, the concern for human health condition is also growing. Body temperature is a vital sign and an important indicator for health monitoring. Thus, temperature sensors are an important tool for monitoring several activities of the human body. However, if these temperature sensors are rigid and large,

\footnotetext{
*Corresponding author: e-mail: linzh@mx.nthu.edu.tw

** Corresponding author: e-mail: ylwang@mx.nthu.edu.tw

https://doi.org/10.18494/SAM.2020.2730
} 
it may cause discomfort. Thus, temperature sensors that are flexible, thin, and easily attachable to different parts of the body or that easily conform to any surface are more desirable. ${ }^{(2-7)}$ Tellurium-based thermoelectric nanomaterials are well known for their high Seebeck coefficient and optimal thermoelectric properties compared with their bulk counterparts. ${ }^{(8,9)}$ Various thermoelectric sensors based on Te nanomaterials have been realized thus far for sensing physical or chemical parameters. ${ }^{(10-14)}$ Nanoparticles (NPs) are emerging to be an excellent choice of materials for thermoelectric temperature sensors. ${ }^{(15,16)}$ However, there has not been much studies that explore the synthesis of Te nanoparticles (Te-NPs) by physical methods. In this work, an ultrathin and flexible self-powered temperature sensor based on the thermoelectric effect was fabricated by RF magnetron sputtering Te-NPs on $5 \times 5 \mathrm{~cm}^{2}$ Al foil. A $1 \times 1 \mathrm{~cm}^{2}$ area was then cut, to both sides of which electrodes were attached to fabricate the sensor. The sensor can detect wide temperature ranges from room temperature to $100{ }^{\circ} \mathrm{C}$ and from room temperature to $0{ }^{\circ} \mathrm{C}$. The output is measured using a stable customized thermal system that can create the temperature gradient accurately. It can detect both hot and cold temperatures with a linear detection range. The sensor can detect the surface temperature accurately owing to its small size, small thickness, and flexibility. It can also detect the body temperature just with a finger touch and can be used to measure various temperature ranges in our daily life activities where flexible sensors are necessary.

\section{Procedure}

\subsection{Growth of Te-NPs}

Te-NPs were deposited in $5 \times 5 \mathrm{~cm}^{2}$ Al foil (99.99\% pure purchased from Alpha Aesar) using a 2" spherical Te sputter target (99.99\% pure) by RF magnetron sputtering at $13.56 \mathrm{MHz}$ and a power of $25 \mathrm{~W}$. Ar was used as the carrier gas and the deposition was carried out for $1 \mathrm{~h}$. The entire sputtering process was carried out at room temperature.

\subsection{Fabrication of self-powered temperature sensor and output measurement setup}

The Te-NP-sputtered Al foil was cut into $1 \times 1 \mathrm{~cm}^{2}$ pieces and contact electrodes were fabricated by applying silver paste on both the Al and Te-NP-sputtered sides. The device was attached to a flexible polyurethane (PU) sheet to make it more robust and further covered with Kapton film for packaging. A very stable self-customized thermal system was used to provide heating and cooling. The thermal system has three stages A, B, and C. These stages could be fixed on top of one another. Only two stages are used for a measuring purpose at a time. Stage A provides heating, stage $\mathrm{B}$ is always kept at constant room temperature, and stage $\mathrm{C}$ provides cooling. For our measurement, the Al side was always in contact with stage B and, for the measurement of the sensor performance for temperatures above room temperature $\left(25-100{ }^{\circ} \mathrm{C}\right)$, stage A was used as a heater that kept the temperature of stage B constant at $25{ }^{\circ} \mathrm{C}$. On the other hand, for measuring temperatures below room temperature $\left(25-0{ }^{\circ} \mathrm{C}\right)$, stage $\mathrm{C}$ was used as a cooler, whereas stage $\mathrm{B}$ was maintained at $25^{\circ} \mathrm{C}$. To measure the output performance of 
the sensor, a low noise voltage preamplifier SR-560 manufactured by Stanford Research was used. The sensing mechanism of the device is based on the thermoelectric effect. The stable thermal system provides a temperature gradient across the two terminals of the device and the uniformly deposited Te-NPs help in maintaining the temperature gradient, resulting in an increase in output voltage with the temperature gradient.

\section{Results and Discussion}

After the fabrication of the thermoelectric sensor, the device performance was tested. Figure 1 shows the overall device schematic and the fabrication process. Figure 1(a) shows the sputtering schematic, where Te is sputtered on a $5 \times 5 \mathrm{~cm}^{2} \mathrm{Al}$ substrate by RF magnetron sputtering at a frequency of $13.56 \mathrm{MHz}$ at room temperature with a chamber pressure of $1.6 e^{-2}$ torr and a power of $25 \mathrm{~W}$. Ar is used as the carrier gas with a flow rate of $6 \mathrm{sccm}$ and the total deposition time is $1 \mathrm{~h}$. The Te-NPs are deposited on the Al substrate to form a flexible device, as shown in Fig. 1(b). They also serve as the bottom electrode, which is always maintained at room temperature. The sensor works on the basis of the thermoelectric mechanism, in which waste heat from the surrounding is used as its source, thus making it self-powered. Figure 1(c) shows the working mechanism, the Te-NP side (i.e., the hot or cooling side) and the Al side (i.e., the constant-temperature side). As the temperature gradient is created across the two sides, the output voltage that keeps increasing linearly with the temperature gradient is generated. The surface morphology of the sputtered Te-NPs was characterized by scanning electron

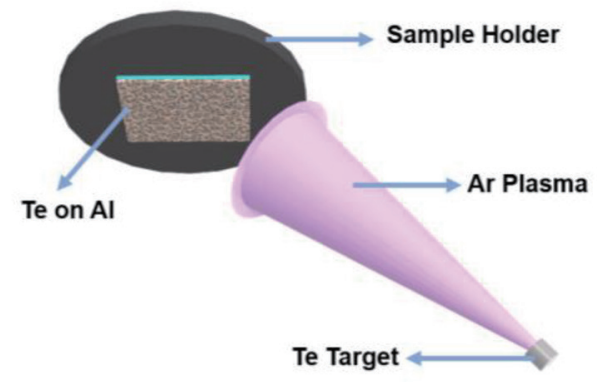

(a)

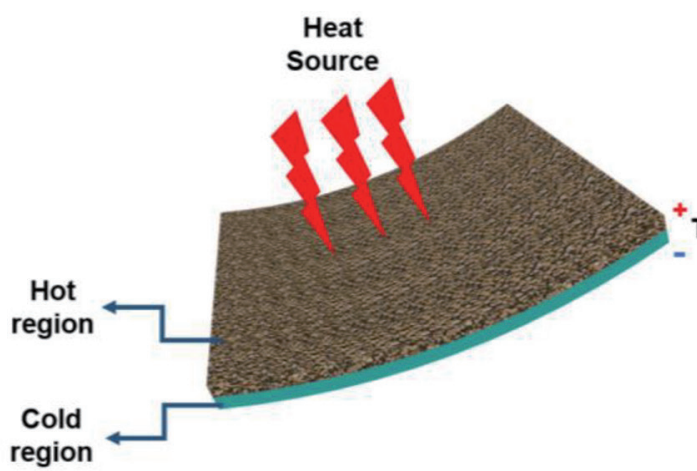

(c)

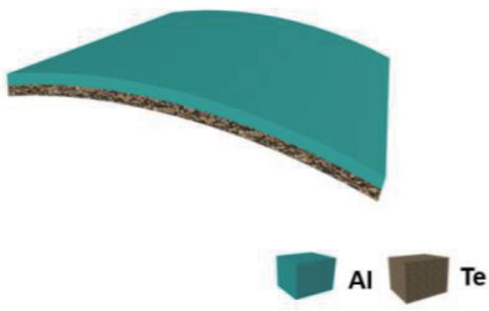

(b)

Thermoelectric voltage principle of thermoelectric sensor. 
microscopy (SEM). Figure 2(a) is a SEM image of Te-NPs, which shows that the surface consists of evenly distributed Te-NPs. The surface includes small and large particles consisting of tiny spherical grains. The particle size was analyzed and calculated using ImageJ software and a number of particles have diameters of around $81 \mathrm{~nm}$ [Fig. 2(b)]. Figure 3(a) shows a SEM image and Fig. 3(b) shows the corresponding energy dispersive spectrometry (EDS)

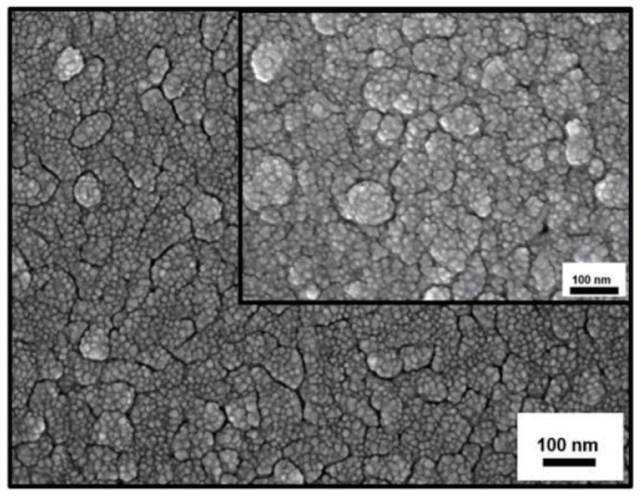

(a)

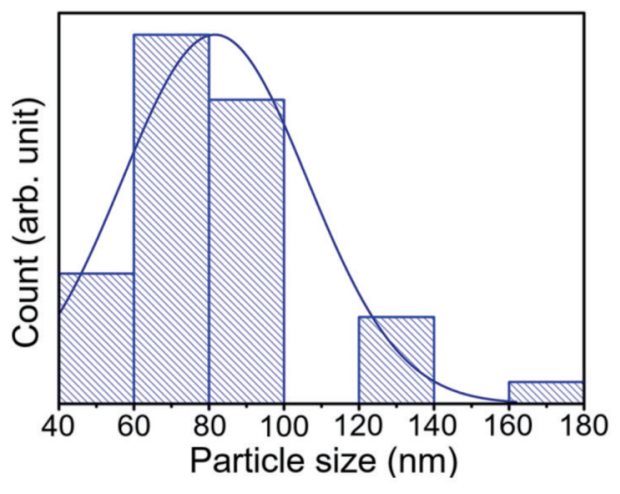

(b)

Fig. 2. (Color online) SEM image of sputtered Te-NPs on Al. (a) High-resolution image with magnified particle image in the inset. (b) Particle size distribution of (a) obtained using ImageJ with gaussian fitting.

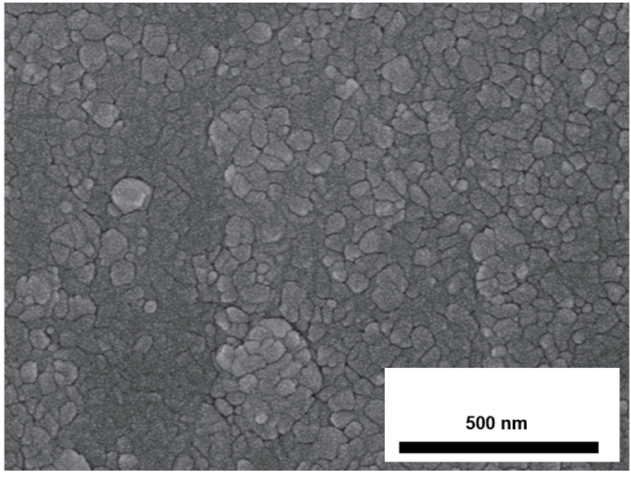

(a)

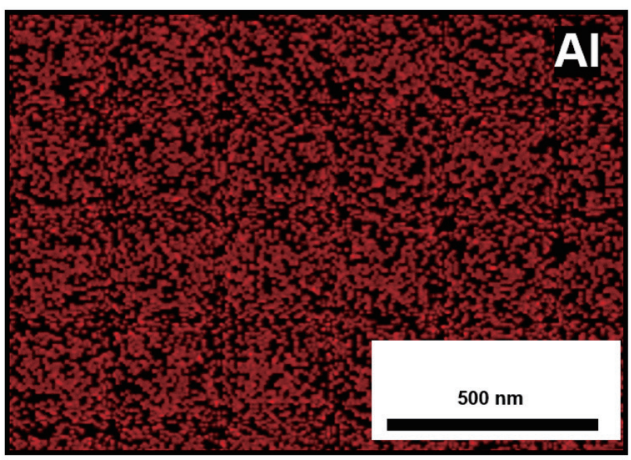

(c)

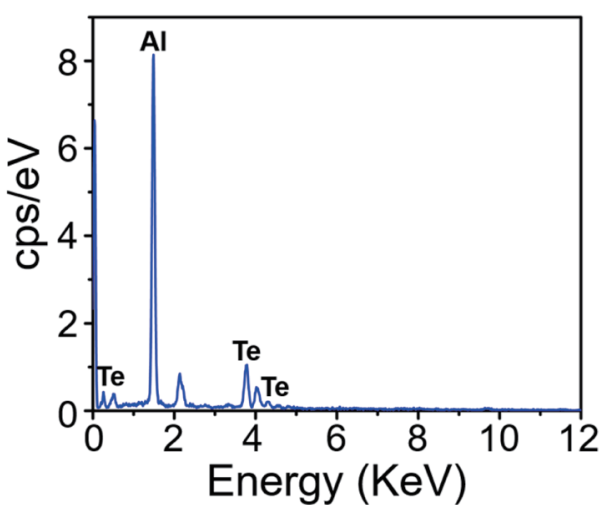

(b)

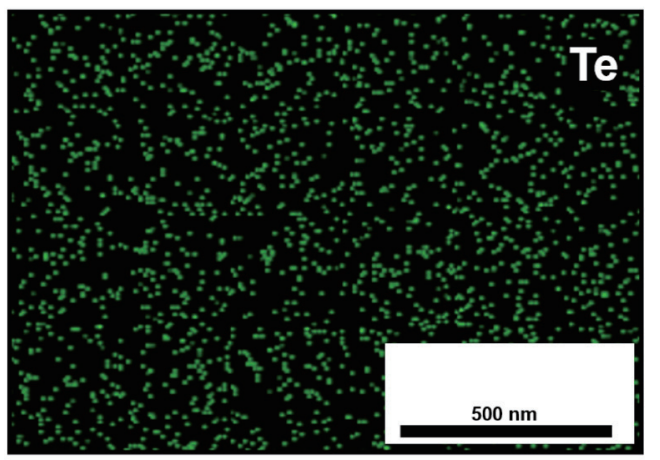

(d)

Fig. 3. (Color online) SEM and EDS mapping. (a) SEM image of Te-NPs on Al, (b) EDS spectrum of (a), (c) elemental mapping of $\mathrm{Al}$, and (d) elemental mapping of Te. 
spectra. Figures 3(c) and 3(d) respectively show the elemental mappings of the substrate Al and the as-prepared Te-NPs. It can be seen that the Te-NPs are evenly distributed over the entire substrate region, which confirms the uniform deposition of the NPs on the substrate. Atomic force microscopy (AFM) measurement shows the surface topography of the Te-NPs on the substrate, which reveals that the Te-NP deposition is continuous [Fig. 4(a)], and the corresponding height profile of the Te-NPs was measured to be around $150 \mathrm{~nm}$ as shown in Fig. 4(b). The X-ray diffraction (XRD) pattern of the as-prepared Te-NPs shows the dominant hexagonal crystalline structure with diffraction peaks at $23.02^{\circ}(101)$ and $40.3^{\circ}(110)$ as shown in Fig. 5. The XRD pattern confirms that the orientation of the Te-NPs is in one direction. The thermoelectric sensor output performance is shown in Fig. 6. Firstly, the Al side of the sensor is maintained at room temperature using stage B of the thermal system and the Te-NP side is exposed to different temperatures ranging from 30 to $100{ }^{\circ} \mathrm{C}$ marked (i) to (xv), where (i) is $30^{\circ} \mathrm{C}$ and continuously increases by $5{ }^{\circ} \mathrm{C}$ in next measurement; thus, measurement (xv) is $100{ }^{\circ} \mathrm{C}$. The output voltage increases with time as shown in Fig. 6(a). The output voltage at $30{ }^{\circ} \mathrm{C}$, i.e., for a temperature gradient of $5{ }^{\circ} \mathrm{C}$ is $0.04 \mathrm{mV}$ and the output voltage measured at $100{ }^{\circ} \mathrm{C}$, i.e., the temperature gradient of $75^{\circ} \mathrm{C}$, is $0.4 \mathrm{mV}$. The output increases linearly with increasing temperature above room temperature and the measured output voltage as a function

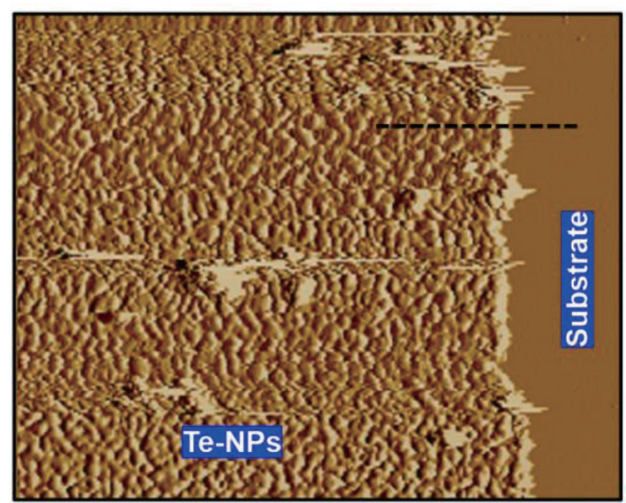

(a)

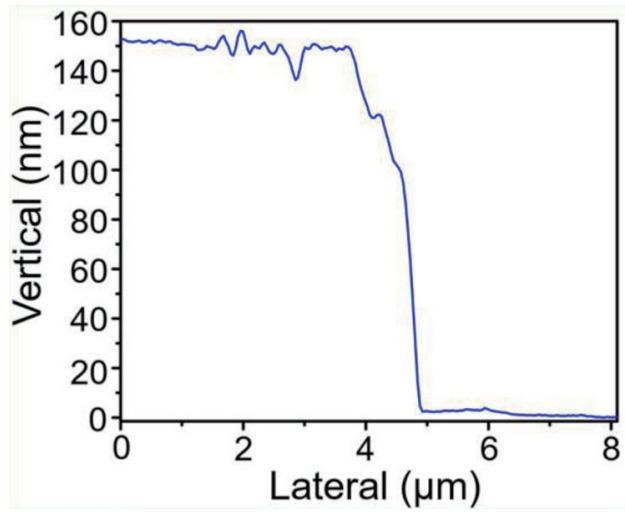

(b)

Fig. 4. (Color online) (a) AFM image of Te-NPs on Al substrate and (b) corresponding height profile of Te-NPs.

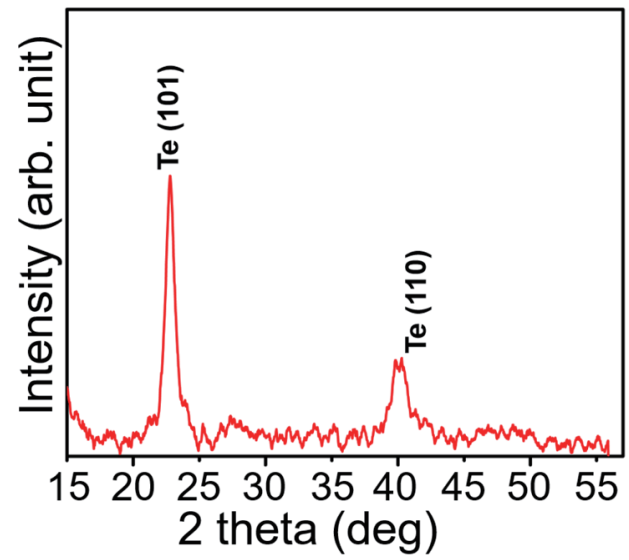

Fig. 5. (Color online) XRD pattern of Te-NPs sputtered on A1. 


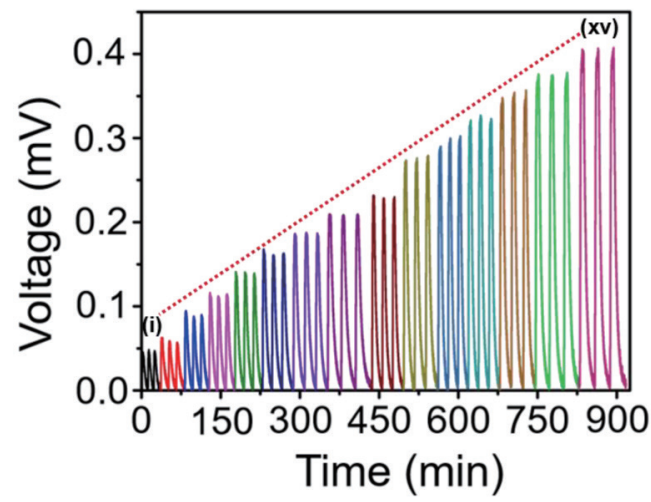

(a)

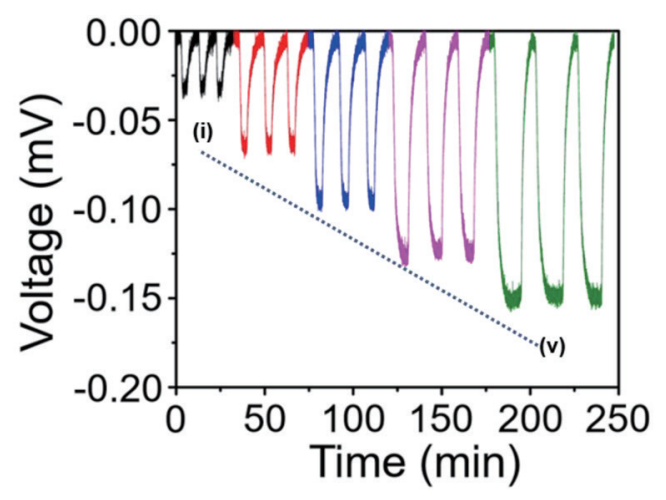

(c)

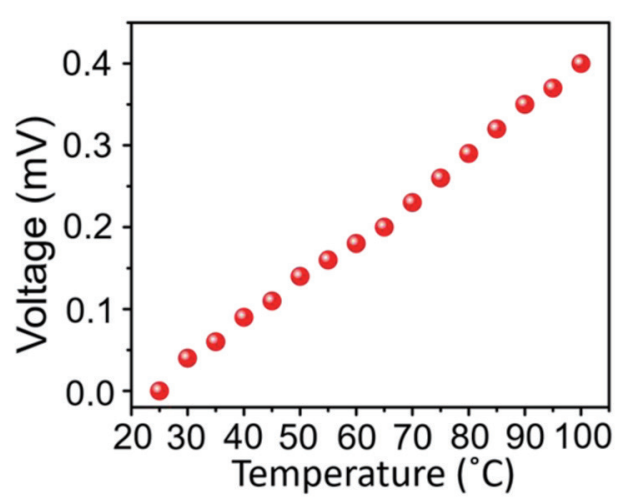

(b)

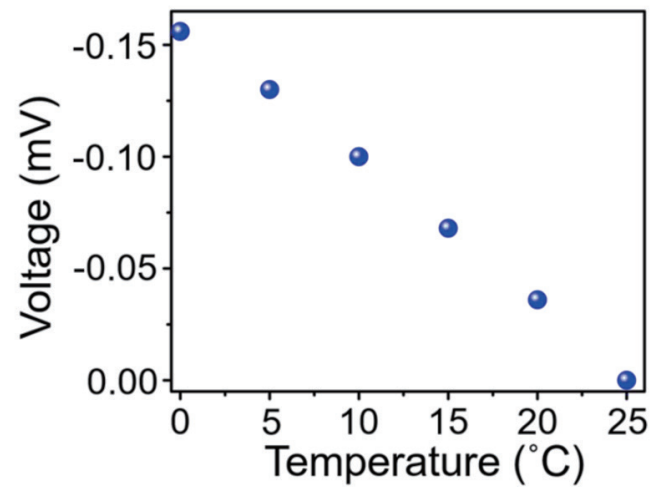

(d)

Fig. 6. (Color online) (a) Output voltage of the thermoelectric sensor from room temperature $\left(25\right.$ to $\left.100{ }^{\circ} \mathrm{C}\right)$ and (b) measured output voltage as a function of temperature gradient. (c) Output voltage of the thermoelectric sensor from room temperature $\left(25\right.$ to $\left.0^{\circ} \mathrm{C}\right)$ and (d) measured output voltage as a function of temperature gradient .

of temperature is shown in Fig. 6(b). In the same manner, the performance of the sensor was measured from room temperature to the freezing point of water, i.e., $0^{\circ} \mathrm{C}$. The output response for this was measured by keeping the $\mathrm{Al}$ side at room temperature using stage $\mathrm{B}$ and the Te-NPs were exposed to temperatures below $25{ }^{\circ} \mathrm{C}$ using the cooling stage $\mathrm{C}$. The obtained output is in the negative direction of the output obtained at temperatures above room temperature. The temperatures are marked (i) to (v) [Fig. 6(c)] where (i) is $20^{\circ} \mathrm{C}$ and the rest of the measurements were obtained by reducing the temperature by $5^{\circ} \mathrm{C}$ up to $0{ }^{\circ} \mathrm{C}$, which is marked (v). The output obtained for a gradient of 5 to $20^{\circ} \mathrm{C}$ ranges from -0.036 to $-0.156 \mathrm{mV}$ and the measured output voltage as a function of time for temperatures below $25^{\circ} \mathrm{C}$ is plotted as shown in Fig. 6(d). The compact size and flexible design of the sensor enable it to measure any surface temperature precisely. A photograph indicating the small device size is shown in Fig. 7(a) and that indicating the device flexibility is shown in Fig. 7(b). Furthermore, the sensor performance in measuring human body temperature with just a finger touch was tested [Fig. 7(c)], and the output response for the touch was determined to be about $0.078 \mathrm{mV}$ [Fig. 7(d)]. Since the human body temperature is around $37^{\circ} \mathrm{C}$, the measured finger touch response was observed to be between the output measured at $35^{\circ} \mathrm{C}(0.062 \mathrm{mV})$ and that measured at $40{ }^{\circ} \mathrm{C}(0.09 \mathrm{mV})$, which shows that the temperature sensor can be used to monitor human body conditions as well as other dayto-day activities in which the temperature sensor plays an important role. 


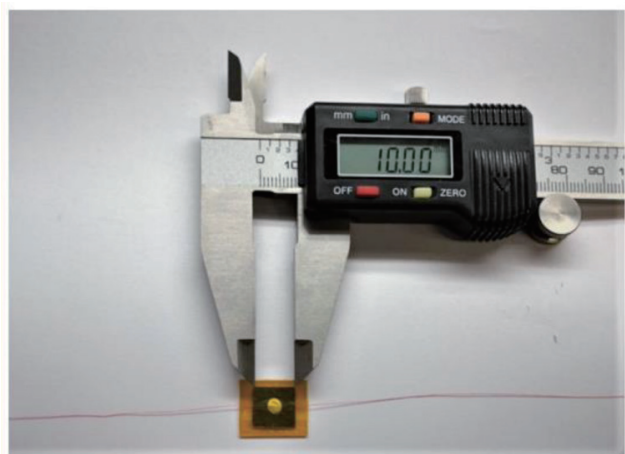

(a)

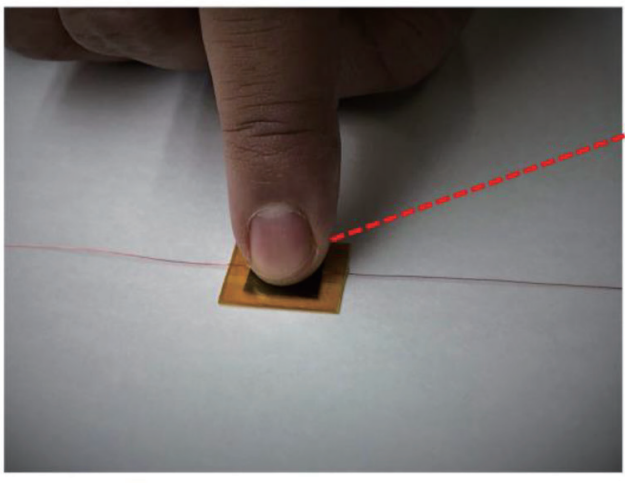

(c)

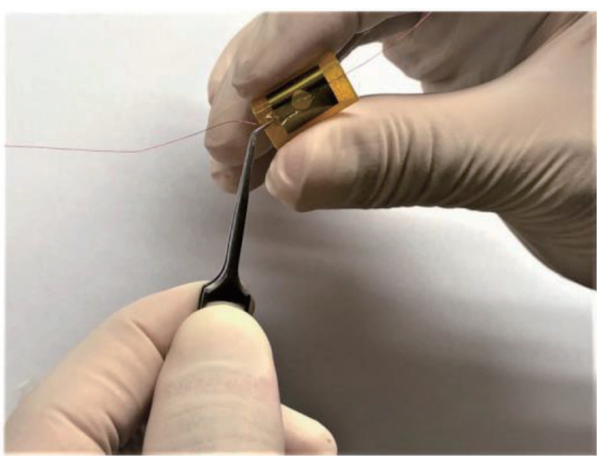

(b)

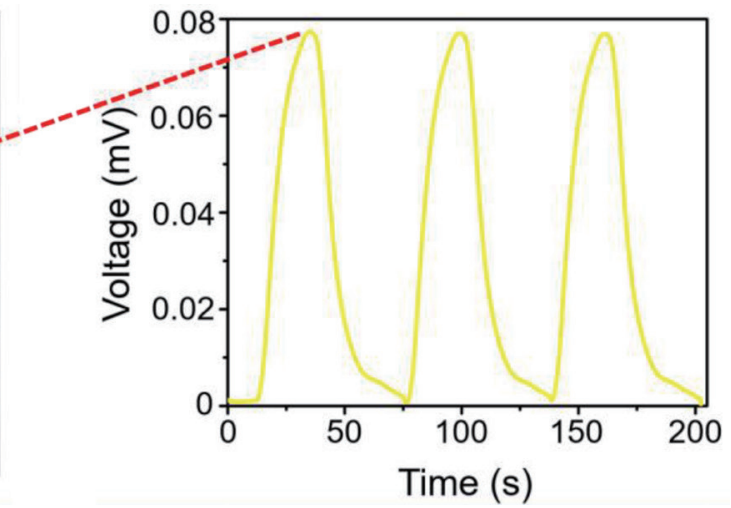

(d)

Fig. 7. (Color online) Photographs of (a) final device $1 \times 1 \mathrm{~cm}^{2}$ size, (b) device flexibility, (c) touching device with index finger, and (d) output response of device to touch and release.

\section{Conclusion}

We fabricated and tested an ultrathin and flexible self-powered temperature sensor that can detect the surface temperature accurately owing to its small size, small thickness, and flexibility. This sensor can also detect wide temperature ranges from room temperature to $100{ }^{\circ} \mathrm{C}$ and from room temperature to $0{ }^{\circ} \mathrm{C}$. For a temperature gradient of $75{ }^{\circ} \mathrm{C}$, the sensor gives an output voltage of $0.4 \mathrm{mV}$. The output is measured using a stable customized thermal system that can create the temperature gradient accurately. The device can detect the body temperature with just a finger touch and can be used to measure various temperature ranges in our daily life activities where flexible sensors are necessary.

\section{References}

1 M. He, Y.-J. Lin, C.-M. Chiu, W. Yang, B. Zhang, D. Yun, Y. Xie, and Z.-H. Lin: Nano Energy 49 (2018) 588. https://doi.org/10.1016/j.nanoen.2018.04.072

2 Z. Cui, F. R. Poblete, and Y. Zhu: ACS Appl. Mater. Interfaces 11 (2019) 17836. https://doi.org/10.1021/ acsami.9b04045

3 D. Zhang, Y. Wang, and Y. Yang: Small 15 (2019) e1805241. https://doi.org/10.1002/smll.201805241

4 B.-U. Hwang: ACS Nano 9 (2015) 8801. https://doi.org/10.1021/acsnano.5b01835

5 W. Zhang, M. Saliba, D. T. Moore, S. K. Pathak, M. T. Horantner, T. Stergiopoulos, S. D. Stranks, G. E. Eperon, J. A. Alexander-Webber, A. Abate, A. Sadhanala, S. Yao, Y. Chen, R. H. Friend, L. A. Estroff, U. Wiesner, and H. J. Snaith: Nat. Commun. 6 (2015) 6142. https://doi.org/10.1038/ncomms7142 
6 B. Wu, Y. Guo, C. Hou, Q. Zhang, Y. Li, and H. Wang: Adv. Funct. Mater. 29 (2019) 1900304. https://doi. org/10.1002/adfm.201900304

7 T. Q. Trung, S. Ramasundaram, B.-U. Hwang, and N.-E. Lee: Adv. Mater. 28 (2016) 394. https://doi. org/10.1002/adma.201670016

8 Y. Yang, Z.-H. Lin, T. Hou, F. Zhang, and Z. L. Wang: Nano Res. 5 (2012) 888. https://doi.org/10.1007/s12274012-0272-8

9 X. Zeng, C. Yan, L. Ren, T. Zhang, F. Zhou, X. Liang, N. Wang, R. Sun, J.-B. Xu, and C.-P. Wong: Adv. Electron. Mater. 5 (2019) 1800612. https://doi.org/10.1002/aelm.201800612

10 T. Y. Hwang, G. M. Go, S. Park, J. Lee, Y. Song, S. Kim, H. B. Cho, and Y. H. Choa: ACS Appl. Mater. Interfaces 11 (2019) 47015. https://doi.org/10.1021/acsami.9b12604

11 Y.-H. Tsao, R. A. Husain, Y.-J. Lin, I. Khan, S.-W. Chen, and Z.-H. Lin: Nano Energy 62 (2019) 268. https:// doi.org/10.1016/j.nanoen.2019.05.032

12 R. Feng, F. Tang, N. Zhang, and X. Wang: ACS Appl. Mater. Interfaces 11 (2019) 38616. https://doi. org/10.1021/acsami.9b11435

13 K. R. Sapkota, P. Lu, D. L. Medlin, and G. T. Wang: APL Mater. 7 (2019) 081103. https://doi. org/10.1063/1.5109899

14 G. Qiu, S. Huang, M. Segovia, P. K. Venuthurumilli, Y. Wang, W. Wu, X. Xu, and P. D. Ye: Nano Lett. 19 (2019) 1955. https://doi.org/10.1021/acs.nanolett.8b05144

15 M. T. Rahman, C.-Y. Cheng, B. Karagoz, M. Renn, M. Schrandt, A. Gellman, and R. Panat: ACS Appl. Nano Mater. 2 (2019) 3280. https://doi.org/10.1021/acsanm.9b00628

16 M. Scheele, N. Oeschler, K. Meier, A. Kornowski, C. Klinke, and H. Weller: Adv. Funct. Mater. 19 (2009) 3476. https://doi.org/10.1002/adfm.200901261

\section{About the Authors}

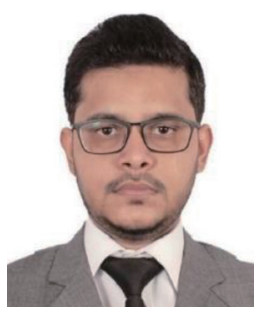

Imran Khan is currently a 3rd year Ph.D. student at the Institute of NanoEngineering and MicroSystems, National Tsing Hua University, under the supervision of Dr. Zong-Hong Lin. His research focuses on the development of highly flexible physical and chemical sensors for in-field environmental monitoring.

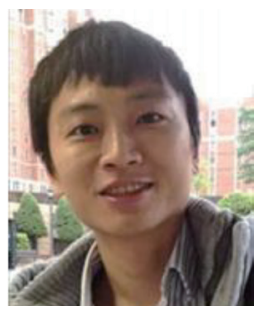

Zong-Hong Lin is currently an associate professor at the Institute of Biomedical Engineering and Department of Power Mechanical Engineering, National Tsing Hua University. His research interests include the understanding of triboelectrification mechanism, the development of active portable sensors for biomedical monitoring, the design of highly efficient catalysts for environmental purification, and self-powered systems with controllable disinfection performance.

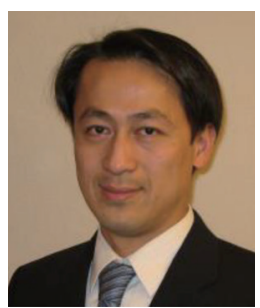

Yu-Lin Wang received his B.S. degree in chemistry from Tunghai University and M.S. degree from National Taiwan University, in 1993 and 1995, respectively. He worked in semiconductor industry from 1997-2006. He received his Ph.D. in materials science and engineering from University of Florida, in 2009. He has been with the Institute of NanoEngineering and Microsystems, National Tsing Hua University, Hsinchu, Taiwan since 2010 as a professor. His current research interests are biosensors, chemical sensors, bioelectronics, medical devices, and semiconductor devices. 